\title{
Status and design of ECRH/CD system of the upgrade of the tokamak T-15
}

\author{
I.N. Roy, I.O. Anashkin, N.A. Kirneva, D.A. Kislov, P.P. Khvostenko, A.V. Melnikov, A.N. Romannikov \\ National Research Centre ‘Kurchatov Institute’, Moscow, Russia, Roy_IN@nrcki.ru
}

The new tokamak T-15MD is under construction in National Research Center «Kurchatov Institute». The tokamak has the following parameters: $\mathrm{R}=1.48 \mathrm{~m}$, $\mathrm{a}=0.67 \mathrm{~m}$ (aspect ratio $\mathrm{A}=2.2), \mathrm{B}=2.0 \mathrm{~T}$, $\mathrm{I}_{\mathrm{pl}}=2.0 \mathrm{MA}$. The poloidal system is capable of realizing the divertor both with single null and double null magnetic configuration and plasma shape with elongation $\mathrm{k}_{95}=$ 1.7-1.9 and triangularity $\delta_{95}=0.3-0.4$. Pulse duration $\Delta \mathrm{t}=10 \mathrm{~s}$, plasma density, $0.5 \cdot 10^{20} \mathrm{~m}^{-3}$, plasma temperature $\mathrm{T}_{\mathrm{i}}(0), \mathrm{T}_{\mathrm{e}}(0), 4-5 \mathrm{keV}$, normalized beta $\beta_{\mathrm{N}}=1.5$.

Preliminary transport analysis with ASTRA code predicts the effective electron and ion heating and transition from $\mathrm{L}$ to $\mathrm{H}$-mode in Ohmic and auxiliary heated regimes. One of the main tasks of experimental study program on $\mathrm{T}-15 \mathrm{U}$ tokamak is the obtaining of physical and technological data for fusion neutron source creation.

$\mathrm{T}-15 \mathrm{MD}$ installation will be equipped with the auxiliary plasma heating and current drive systems $\left(\mathrm{P}_{\mathrm{aux}}>14 \mathrm{MW}\right)$. The NBI system will consist of three hydrogen NB injectors $6 \mathrm{MW}$ total power at pulse duration up to $30 \mathrm{~s}$. The ECRH and CD system will consist of eight gyrotrons more then $8 \mathrm{MW}$ total RF launched power at pulse duration up to $30 \mathrm{~s}$. The $\mathrm{LHH}$ and CD system will be able to launch $4 \mathrm{MW} \mathrm{RF}$ power with $30 \mathrm{~s}$ duration. The ioncyclotron heating system will consist of 3 generators, $30 \div 65 \mathrm{MHz}, 2.5 \mathrm{MW}$, which will allow to inject $6 \mathrm{MW}$ power with pulse duration up to $10 \mathrm{~s}$.

Up to date the components of the magnetic system and vacuum chamber of T-15MD are manufactured. Next two years plan is to perform a test assembly at the factory site, disassemble, deliver it to the Kurchatov Institute site, perform the final assembly and connect to all the systems (electrical, vacuum, water-cooling, etc.).

At the same time, the design of the ECR system should be performed.

The gyrotron auxiliary heating and current drive system should allow to provide: ECRH; ECCD; Control of MHD instabilities by ECRH; ECRH pre ionization; Electron Bernstein Wave Heating.

To fulfill the above tasks, was selected the system of two with a frequency of $82.6 \mathrm{GHz}$ and six gyrotrons with a frequency of $105 \mathrm{GHz}$. The power generated by each of the gyrotrons must be at least $1 \mathrm{MW}$, the preferred value is $1.5 \mathrm{MW}$ in Gaussian beam. Pulse duration from 20 to 30 s. Efficiency not less 50\% (gyrotrons equipped with depressed collectors).

The magnetic systems will consist of: the main cryogenic magnets (liquid Helium free, with remotely located cryocompressor), cathode coils, collector sweeping coils.

A new location was chosen for the new ECR system (Fig. 1.). Thus, to minimize the effect of magnetic fields of the tokamak. Calculations showed that at the location of the gyrotrons the radial component of the magnetic field in all regimes does not exceed $1.0 \mathrm{G}$.

The evacuated corrugated waveguide transmission line $(\varnothing 63.5 \mathrm{~mm})$ will transport the RF power. The length of the transmission lines will be from 27 to $42 \mathrm{~m}$. They will also contain:

- Matching Optic Unit,

- RF wave polarizer providing linear or elliptical wave polarization rotation,

- waveguide switch, directing the power to the calorimetric load or to the tokamak.

- $\quad$ short pulse (up to $0.1 \mathrm{~s}$ ) calorimetric measuring load,

- second waveguide switch, directing the power to the terminal load or to the tokamak

- full pulse terminal load,

- waveguide mechanical break (the tokamak will move $15 \mathrm{~mm}$ in radial and vertical directions due to thermal expansion),

- waveguide DC break $(1 \mathrm{kV})$,

- RF vacuum valve,

- RF launcher. Two fully articulating launchers for ECRH/CD at weak field side will operate with four RF beams each. Two launchers at strong field side will operate with one RF beam each.

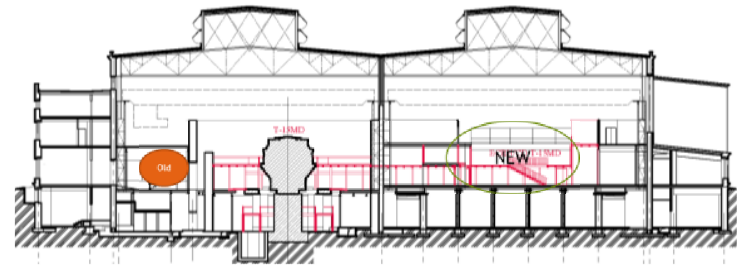

Fig. 1. Old and new location of ECR in building of T-15

The system will be equipped with the low-voltage power supplies necessary for the operation of the gyrotron: superconducting magnet power supply, collector sweep coil power supply, collector dc coil power supply, ion pump power supply, cathode filament power supply, gun coil power supply.

The water cooling system of the gyrotron and the critical elements of the transmission line should be provided.

All the equipment must be remotely controlled. Hardware-software system will be created for monitoring, control, protection and data acquisition.

Currently Kurchatov Institute, there are only two high voltage power supplies can operate with parameters required for T-15MD gyrotrons. Updating or creation of the new main high voltage power supplies is necessary to fulfill the tasks of the ECRH/CD system

This work was partially supported by ROSATOM State Corporation, contract H.4x.241.9Б.17.1011.

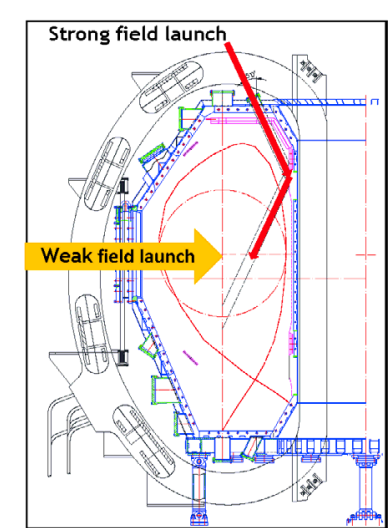

Fig. 2. Vertical cross-section of the T-15MD. 